\title{
Immer den Durchblick bewahren
}

\begin{abstract}
— Wissenschaftler und Studenten müssen sich häufig mit Zahlen und Fakten verschiedener Studien beschäftigen. Wie aber behält man in diesem Datendschungel den Durchblick?

ATLAS.ti ist eine der führenden Softwares, die bei qualitativer Methodik schon seit vielen Jahren in der Gesundheitsforschung eingesetzt wird. Denn sie liefert komplexe Funktionen bei einfacher Bedienung und hilft somit bei der Analyse und Organisation "weicher" Daten bei wissenschaftlichen Studien, Aktenauswertung und Gutachtenevaluation. ATLAS.ti hilft, die in Datenmengen verborgenen komplexen Phäno-
\end{abstract}

mene herauszuarbeiten und produktiv nutzbar zu machen. Dazu bietet es eine ausgesprochen leistungsfähige und intuitive Arbeitsumgebung, die sich der Arbeitsweise des Benutzers ideal anpasst. Die Software unterstützt bei:

_ Klassischen Grounded Theory Studien über chronische Krankheiten

_ Studien zur Zusammenarbeit von Pflegekräften, Ärzten, Psychotherapeuten, Logopäden

_ Studien zur Verbesserung der Arbeit mit Demenzpatienten

_ Studien über die Einbeziehung von Familienmitgliedern in eine Therapie

\section{Experten im Gespräch \\ Plötzlich Pflegefall}

— Wenn ein Mensch Pflege braucht, gibt es viele Fragen. Häufig ist die ganze Familie gefordert, wenn ein Angehöriger plötzlich zum Pflegefall wird. Wo finden Betroffene schnell Unterstützung? Was gilt es bei Anträgen \& Co zu beachten? Welche Unterstützung für die Angehörigen gibt es eigentlich?

Experten aus Berlin und Brandenburg stellen sich den Fragen, geben praktische Tipps und erklären, welche Änderungen das Pflegestärkungsgesetz II mit sich bringt. Abgerundet wird die Gesprächsrunde - eine kostenlose Veranstaltung der SBK Siemens Betriebskrankenkasse - mit einem Ausblick auf das Pflegestärkungsgesetz III.

SBK-Talk für Pflegebedürftige, Angehörige und Pflegekräfte.

Termin: 20. Januar 2017, 17.00 - 19.00 Uhr

Informationen und Buchungen unter:

www.gesundheitskongresse.de 